\title{
Programa Hospital Seguro para a Pessoa Idosa: estudo observacional do impacto na redução de declínio funcional
}

\author{
Safe Hospital Program for the Elderly Person: \\ an observational study on the impact in \\ reducing functional decline
}

\section{Programa Hospital Seguro para Personas Ancianas: estudio observacional de impacto en la reducción del deterioro funcional}

Daniel Apolinario 1

Siomara Tavares Fernandes Yamaguti 1

Adriana Fatina Dutra 1

Enilda Maria de Sousa Lara 1

Rita de Cassia Pires Coli 1

Bernardete Weber 1

\section{Resumo}

Ao ser hospitalizado, o idoso apresenta maior risco de complicações e declínio funcional. O presente estudo avaliou os resultados do Programa Hospital Seguro para a Pessoa Idosa. A intervenção foi realizada em um hospital filantrópico da cidade de São Paulo, Brasil, por meio de uma estratégia de implantação estruturada em 10 passos, incluindo rastreio de fragilidade, avaliação multidimensional, plano terapêutico singular, implementação de protocolos e estratégias de gestão de alta. Foram analisadas ao longo de dois anos 865 internações de idosos frágeis. Indicadores de processo e de resultado foram comparados entre o primeiro ano (período de implementação) e o segundo ano (período de consolidação). A comparação dos indicadores entre os dois períodos revelou que o programa melhorou os processos assistenciais fundamentais na qualidade do cuidado para essa população, incluindo reabilitação motora iniciada nas primeiras 72 horas (74,1 vs. 84,3\%; $p<0,001)$, triagem de risco de broncoaspiração (38,5 vs. 82, 8\%; $p<0,001)$ e suplementação nutricional oral (55, 6 vs. 76,4\%; $p<0,001)$. A taxa de declínio funcional, que era 17,2\% no ano de implantação, caiu para 11,7\% no ano de consolidação, com diferença significativa após ajuste em modelo multivariável $(p=0,009)$. Em conclusão, desenvolvemos um programa factível para a realidade dos hospitais brasileiros e que pode ser reproduzido em outros centros usando-se uma metodologia sistematizada de implantação. Os resultados relativos aos indicadores de processos assistenciais e desfechos clinicos parecem promissores. A disseminação dessa iniciativa deve ser considerada no planejamento das politicas de saúde pública para a rede hospitalar.

Hospitalização; Fragilidade; Atividades Cotidianas; Melhoria de Qualidade; Idoso

\author{
Correspondência \\ D. Apolinario \\ Rua Marc Chagall 397, Torre Curió, apto. 21, São Paulo, SP \\ 05036-170, Brasil. \\ daniel_apolinario@yahoo.com \\ 1 Associação Beneficente Síria, Hospital do Coração, São Paulo, \\ Brasil.
}




\section{Introdução}

Como resultado da rápida transição demográfica observada no Brasil, os idosos são responsáveis por uma proporção cada vez maior das internações hospitalares. Em 2019, os indivíduos com 60 anos ou mais representavam $15,7 \%$ da população brasileira, mas foram responsáveis por $26,4 \%$ das internações no Sistema Único de Saúde (SUS) 1,2. Além de uma maior taxa de internações, os idosos apresentam maior tempo de permanência. No ano de 2019, o tempo médio de permanência foi de 6,5 dias entre os idosos, em comparação a 4,8 dias entre pacientes com até 59 anos 2 . O custo atribuído a internações de pacientes idosos no SUS, que em 2009 representava 27,7\% do total, saltou para 35,8\% em 20192. Essa tendência tende a acelerar nos próximos anos e impõe à rede hospitalar o desafio premente de desenvolver linhas de cuidado mais eficientes para as necessidades específicas dessa população 3 .

Entre os conceitos importantes no planejamento da atenção ao idoso hospitalizado destaca-se a fragilidade, síndrome multifatorial caracterizada pelo desenvolvimento cumulativo de déficits e comorbidades, que em sua evolução pode cursar com a redução da força muscular, comprometimento da marcha e dependência funcional 4,5. A fragilidade está associada à redução das reservas fisiológicas, fazendo com que o idoso tenha um maior risco de agudizações ${ }^{6}$. Ao ser hospitalizado, o idoso frágil apresenta uma recuperação mais lenta e maior risco de complicações secundárias, que não estão relacionadas diretamente ao motivo inicial da internação 7.

Entre as complicações secundárias frequentes nos idosos frágeis hospitalizados estão as reações adversas a medicamentos, deterioração do estado nutricional, queda, lesão por pressão, pneumonia aspirativa e delirium 8,9. Muitos desses indivíduos sofrem contenção mecânica, medida que tem sido banalizada para facilitar o cuidado 10 . O imobilismo durante a internação é acompanhado de uma rápida perda de massa muscular e pode resultar em dependência funcional, complicação que afeta a qualidade de vida do indivíduo e aumenta as demandas de cuidado após a alta 11,12.

Em uma revisão sistemática, Cunha et al. 13 observaram que a proporção de idosos frágeis que não recuperou o desempenho funcional prévio após uma internação variou entre 8,5\% e $48 \%$ em diferentes hospitais, a depender da qualidade das medidas preventivas utilizadas. Estratégias para prevenir complicações, promover a mobilização e oferecer recursos de reabilitação precocemente têm sido bem sucedidas na função de evitar complicações secundárias e reduzir o declínio funcional associado à internação 14,15 .

Alguns programas foram desenvolvidos para apoiar as instituições hospitalares na qualificação do atendimento ao idoso hospitalizado. Entre essas iniciativas destaca-se o Hospital Elder Life Program (HELP), com foco na prevenção do delirium e do declínio funcional 16 . Desenvolvido originalmente na Universidade de Yale (Estados Unidos) em 1993, atualmente o programa HELP já foi implantado em mais de 200 hospitais e reúne evidências consistentes de efetividade na melhoria de desfechos clínicos e na redução de custos 17 . Outros programas foram desenvolvidos nessa mesma linha, como o Nurses Improving Care for Healthsystem Elders (NICHE) 18, Better Outcomes for Older adults through Safe Transitions (BOOST) 19 e o Age-Friendly Health System (AFHS) 20.

Nesses programas, o hospital que deseja melhorar os resultados relacionados à internação de pacientes idosos adere a um pacote de melhorias, implementa protocolos baseados em evidências, integra fóruns de gestores para a troca de experiências, tem acesso a recursos de capacitação e passa a utilizar indicadores padronizados para monitorar a qualidade dos serviços. No entanto, os programas existentes no cenário internacional permanecem restritos aos países desenvolvidos e não têm sido implantados de forma consistente nos países em desenvolvimento. Entre as características desses programas que dificultam a inclusão dos hospitais em países como o Brasil podemos citar a necessidade de investimento financeiro para a adesão, a disponibilidade dos materiais de capacitação apenas em inglês e a exigência de profissionais especializados em Geriatria e Gerontologia para desenvolver as atividades propostas.

Com o objetivo de oferecer uma iniciativa compatível com a disponibilidade de recursos materiais e humanos dos hospitais brasileiros, desenvolvemos o Programa Hospital Seguro para a Pessoa Idosa, um modelo estruturado de melhoria dos processos assistenciais com foco na prevenção de complicações secundárias em idosos frágeis. A iniciativa é compatível com o conceito de segurança do paciente da Organização Mundial da Saúde, definido como redução dos riscos de danos desnecessários associados à assistência à saúde até um mínimo aceitável 21. É uma proposta multidisciplinar que, em vez 
da especialização vertical e da segmentação, propõe a disseminação horizontal do conhecimento e a construção de uma rede de segurança que permeia o percurso do paciente idoso no hospital. Com um método de implantação estruturado passo a passo, o programa baseia-se em um conjunto de ações que inclui reorganização de fluxos, capacitação de profissionais, implementação de protocolos e monitoramento de indicadores. Este estudo tem como objetivo descrever o Programa Hospital Seguro para a Pessoa Idosa e avaliar os resultados iniciais da sua implantação em um hospital filantrópico da cidade de São Paulo.

\section{Métodos}

\section{Desenvolvimento do programa}

O Programa Hospital Seguro para a Pessoa Idosa foi desenvolvido entre 2017 e 2018 com o objetivo de constituir um modelo estruturado de melhoria da atenção ao idoso hospitalizado, reduzindo assim os danos desnecessários comumente associados à internação. O programa foi desenvolvido por uma equipe multidisciplinar composta por gestores da área hospitalar, especialistas na área de envelhecimento, especialistas em políticas públicas de atenção ao idoso e profissionais com cargos de coordenação em ambiente hospitalar das áreas de Enfermagem, Fisioterapia, Fonoaudiologia, Nutrição, Psicologia e Farmácia Clínica.

Em uma etapa inicial, a equipe realizou visitas a hospitais que já tinham iniciativas nessa área. Em seguida, foi conduzida uma revisão da literatura pertinente a programas existentes em outros países. Em destaque, o programa HELP foi utilizado para guiar as ações de prevenção de declínio funcional e delirium, o programa BOOST inspirou ações de transição do cuidado e o programa NICHE serviu como referência para definir os papéis dos profissionais de enfermagem 16,17,18,19. Foram usadas ainda na construção do modelo algumas propostas do Projeto Idoso Bem Cuidado da Agência Nacional de Saúde Suplementar, e contemplados os pré-requisitos do Selo Hospital Amigo do Idoso da Secretaria de Saúde do Estado de São Paulo 22,23.

$\mathrm{Na}$ próxima etapa foram realizados grupos focais com idosos hospitalizados e seus cuidadores, visando a identificar valores e prioridades. Nessa oportunidade ficou clara a preocupação com a possibilidade de deterioração da qualidade de vida e perda de independência funcional persistente no período pós-alta, o que determinou a intensificação de ações para prevenir complicações com o impacto funcional. Nesse período, foram realizados ainda pilotos utilizando os modelos de ciclo rápido de melhoria propostos pelo Instituto para a Melhoria da Saúde (Institute for Healthcare Improvement - IHI, Estados Unidos) para testar e aprimorar as principais rotinas de avaliação e de intervenção 24.

Após a definição das ações que deveriam compor o programa, a estratégia de implantação foi organizada em 10 passos sequenciais para facilitar o planejamento e garantir que a iniciativa pudesse ser reproduzida em outros hospitais. Os 10 passos do programa estão descritos de forma resumida no Quadro 1. Destacam-se os passos de número quatro a sete, que promovem mudanças substanciais nos processos de cuidado: (4) rastreio de fragilidade para selecionar uma população com maior risco de complicações e maior potencial de benefício; (5) avaliação multidimensional para identificar pontos de vulnerabilidade e necessidades específicas; (6) estratégia de gestão de casos baseada no plano terapêutico singular; e (7) implementação de protocolos de prevenção e manejo das síndromes geriátricas mais frequentes, incluindo quedas, lesão por pressão, broncoaspiração, delirium, desnutrição, imobilismo, iatrogenia medicamentosa e contenção mecânica.

Para facilitar a reprodutibilidade e a disseminação do programa foram desenvolvidos materiais de apoio com o financiamento do Programa de Apoio ao Desenvolvimento Institucional do SUS (PROADI-SUS), incluindo guia de implementação para gestores, caderno de capacitação para profissionais da linha assistencial, protocolos de prevenção de síndromes geriátricas e ambiente virtual de aprendizagem com vídeo aulas e hipertextos. Todos os materiais de apoio para a implantação do Programa podem ser obtidos sem custo por intermédio de contato com os autores. 
Quadro 1

Os 10 passos do processo da implantação do Programa Hospital Seguro para a Pessoa Idosa. São Paulo, Brasil, $2018 / 2019$.

\section{CONSTITUIÇÃO DO COMITÊ GESTOR E DO GRUPO DE TRABALHO}

O comitê gestor tem a função de definir o modelo geral, indicar o grupo de trabalho, dar apoio estratégico e supervisionar o programa. Deve ser composto por duas ou mais lideranças, sendo pelo menos uma com perfil administrativo e uma com perfil assistencial. O grupo de trabalho formula um projeto operacional de implantação, executa as ações planejadas e monitora os indicadores. Deve ser composto por pelo menos 6 profissionais representando diferentes áreas e setores.

\section{DIAGNÓSTICO DOS PROCESSOS E DOS RECURSOS DISPONÍVEIS}

Esta etapa permite reconhecer as oportunidades de melhoria e dimensionar os recursos necessários para a implantação do programa. Fornece ainda dados importantes para o desenvolvimento de um modelo customizado, que seja compatível com o perfil da instituição. Consiste no levantamento de dados epidemiológicos relativos aos pacientes idosos, revisão dos indicadores e pilotos para rastrear a prevalência de algumas condições (p.ex.: investigar a proporção de idosos frágeis nas unidades de internação).

\section{DEFINIÇÃO DO MODELO GERAL A SER IMPLANTADO}

Refere-se ao delineamento do Programa em seus fluxos principais, definido pelo comitê gestor por meio de cinco questões: (1) O hospital contará com um ambiente específico para idosos frágeis (Unidade Geriátrica)? (2) Em que ambientes/unidades o Programa será implantado? (3) A seleção

dos participantes (p.ex.: rastreio de fragilidade) será realizada em que local, em que momento e por qual profissional? (4) De que forma os participantes do programa serão identificados (p.ex.: carimbo no prontuário, sinalização no leito, pop-up no prontuário eletrônico)? (5) Quais são os prazos para a implantação de cada etapa no cronograma?

\section{SELEÇÃO DA POPULAÇÃO-ALVO}

A presença de fragilidade é utilizada como critério de elegibilidade para o programa, pois permite a seleção de pacientes com maior risco de complicações e direciona os recursos para a população com maior potencial de benefício. Um questionário de rastreio de fragilidade é aplicado em todos os indivíduos com 60 anos ou mais e um ponto de corte é selecionado para definir a elegibilidade.

\section{AVALIAÇÃO MULTIDIMENSIONAL DA PESSOA IDOSA (AMPI)}

Trata-se de um processo diagnóstico multidisciplinar que fornece um perfil de capacidades intrínsecas e de vulnerabilidades, permitindo uma visão global das prioridades que auxilia na formulação do projeto terapêutico individual, na definição de metas e no monitoramento. Entre os componentes obrigatórios na composição da AMPI hospitalar estão: (1) rastreio cognitivo; (2) rastreio de delirium; (3) sintomas depressivos;

(4) atividades da vida diária; (5) mobilidade; (6) risco nutricional; (7) risco de broncoaspiração; (8) rastreio de medicações potencialmente inapropriadas para idosos; (9) barreiras de comunicação; e (10) suporte social.

\section{GESTÃO DE CASOS E ESTRATÉGIAS DE ATUAÇÃO DAS EQUIPES}

Um enfermeiro exerce a função de gestor de casos. Esse profissional formula o plano terapêutico, aciona protocolos e equipes, acompanha a evolução do paciente, atua como elo de integração entre equipes e ambientes, realiza intervenções educativas, participa do planejamento de alta e coordena transições do cuidado. As demais equipes atuam de acordo com os critérios preestabelecidos (p.ex.: equipe de Fonoaudiologia é acionada quando o rastreio para o risco de broncoaspiração for positivo). Uma ronda multidisciplinar é realizada para discutir casos de pacientes incluídos no Programa e um formulário interdisciplinar é utilizado para o compartilhamento das informações de interesse comum entre as equipes.

\section{DESENVOLVIMENTO E APRIMORAMENTO DE PROTOCOLOS}

Cinco protocolos multidisciplinares de prevenção e manejo das síndromes geriátricas são desenvolvidos ou aprimorados com base em modelos desenvolvidos por especialistas, que podem ser adaptados às peculiaridades do hospital: (1) prevenção de quedas; (2) prevenção de lesão por pressão; (3) prevenção de broncoaspiração; (4) prevenção, detecção e manejo do delirium; (5) uso racional da contenção mecânica. De forma adicional, podem ser considerados ainda protocolos de estímulo à mobilidade, rastreio de medicações inapropriadas para idosos e abordagem multidisciplinar do risco nutricional.

\section{ESTRATÉGIAS DE EDUCAÇÃO PERMANENTE E ENGAJAMENTO}

As ações educativas têm o objetivo de capacitar profissionais de diferentes áreas para que tenham uma visão global dos processos de atenção ao idoso. Todos os profissionais da linha assistencial devem ser capazes de interpretar os resultados do questionário de rastreio de fragilidade e da AMPI. Devem ainda dominar conhecimentos básicos sobre oito complicações de grande relevância no ambiente hospitalar: (1) queda; (2) lesão por pressão; (3) broncoaspiração; (4) delirium; (5) contenção mecânica; (6) desnutrição; (7) iatrogenia medicamentosa; e (8) declínio funcional. Entre os recursos utilizados estão um caderno de capacitação, vídeo aulas, infográficos, treinamento de pequenos grupos in loco, oficinas de

\section{GESTÃO DE ALTA E INTEGRAÇÃO COM A REDE} sensibilização e reuniões para discussão de casos.

Um Núcleo de Gestão de Altas é formado para identificar os fatores relacionados à permanência prolongada específicos da instituição, implementar ferramentas para identificar risco de longa permanência, mapear os recursos de desospitalização disponíveis na rede, e desenvolver um programa de planejamento de alta com ênfase na transição do cuidado.

10. MONITORAMENTO DE INDICADORES

Os indicadores são fundamentais para que a instituição monitore os seus progressos. Os indicadores básicos monitorados neste programa são: tempo de permanência; densidade de incidência de queda; incidência de lesão por pressão; incidência de pneumonia por broncoaspiração; incidência de delirium; taxa de contenção mecânica. 


\section{Ambiente e participantes}

O trabalho foi conduzido em um hospital filantrópico na cidade de São Paulo, que conta com 267 leitos operacionais e atende a uma clientela predominantemente idosa. No período em que o estudo foi conduzido, os pacientes com 60 anos ou mais foram responsáveis por $54 \%$ das internações e ocuparam 63\% dos leitos/dia disponíveis.

O estudo compreende o período de janeiro de 2018 a dezembro de 2019. Ao longo do primeiro ano as ações previstas foram iniciadas em passos sucessivos e ampliadas progressivamente. Ao final do primeiro ano todos os processos previstos já haviam sido iniciados e todas as unidades de internação já tinham sido incluídas. No ano seguinte, os processos já iniciados foram aprimorados e consolidados. Embora a implantação tenha sido realizada de forma progressiva e sem ruptura, o primeiro período (2018) foi denominado de "ano de implementação" e o período seguinte (2019) "ano de consolidação", de forma que os desfechos de interesse pudessem ser comparados de forma evolutiva.

O principal critério de inclusão no trabalho coincide com o critério de elegibilidade para a participação no Programa, definido como a presença de fragilidade e indicado por meio de um escore 3 ou maior na escala FRAIL, aplicada pelo enfermeiro da unidade no momento da admissão 25,26. A escala apresenta cinco itens que avaliam marcha, força, fadiga, perda ponderal involuntária e multimorbidade. Para aplicar a escala FRAIL, todos os enfermeiros da instituição foram convocados para o treinamento presencial obrigatório com duração de uma hora. O treinamento utilizou metodologia interativa baseada em casos clínicos. Nos itens que avaliam marcha, força e fadiga, os profissionais foram orientados a considerar o estado prévio, anterior ao processo de agudização, tomando duas semanas atrás como ponto de referência.

Foram excluídos os pacientes com tempo de permanência menor que 72 horas, que sofreram óbito durante a internação ou com escore < 5 no Índice de Barthel. Esse Índice avalia as atividades básicas da vida diária, incluindo alimentar-se, tomar banho, manter a higiene pessoal, vestir-se, manter o controle urinário e fecal, utilizar o vaso sanitário, fazer transferências, deambular e subir escadas 27. Pacientes que tinham menos de 5 pontos no Índice de Barthel da linha de base foram excluídos por já apresentarem dependência funcional grave e não estarem expostos ao desfecho primário do estudo, definido como redução de 5 pontos ou mais no Índice de Barthel.

Os testes, questionários e escalas aplicados como rotina na avaliação multidimensional da admissão foram usados para descrever a amostra e realizar ajustes nos modelos multivariáveis, incluindo avaliação cognitiva com o 10-point Cognitive Screener (10-CS) 28, rastreio de delirium com o Confusion Assessment Method (CAM) 29, avaliação do desempenho de membros inferiores com a Short Physical Performance Battery (SPPB) 30 e avaliação nutricional com a Mini-Avaliação Nutricional (MAN) 31. A circunferência da panturrilha foi medida com trena antropométrica para fornecer uma estimativa da massa muscular, considerada reduzida quando menor que $31 \mathrm{~cm}$. A força de preensão palmar foi medida com dinamômetro hidráulico Saehan.

\section{Desfechos}

O desfecho primário do estudo foi definido como declínio funcional associado à internação e indicado pela variação evolutiva no Índice de Barthel. Neste trabalho, utilizamos a versão do Índice com pontuação que varia de 0 a 20 e na qual a perda de 5 pontos é considerada significativa de acordo com a metodologia de diferença mínima detectável 32,33. Na análise desse indicador, a pontuação da linha de base, representando como o paciente era em casa duas semanas antes da internação, foi comparada à pontuação do índice obtido em uma entrevista telefônica realizada 30 dias após a alta.

Como desfechos secundários foram analisadas sete complicações comuns entre idosos hospitalizados, definidas neste estudo de forma dicotômica: (1) ocorrência de queda durante a internação (uma ou mais); (2) incidência de lesão por pressão nova durante a internação (estágio 2 ou maior); (3) episódio de broncoaspiração sintomática; (4) transferência não planejada para a unidade de terapia intensiva; (5) institucionalização (alta para a instituição de longa permanência); (6) internação prolongada (maior que 30 dias); e (7) reinternação precoce (em até 30 dias após a alta). 
Para avaliar as mudanças na qualidade do atendimento foram monitorados quatro indicadores de processo de grande relevância na atenção ao idoso frágil: (1) rastreio de medicações potencialmente inapropriadas para idosos pelos critérios de Beers e o respectivo contato com o médico assistente 34 , realizado pela equipe de Farmácia Clínica dentro de 72 horas da admissão; (2) reabilitação motora iniciada pela equipe de Fisioterapia dentro de 72 horas da admissão; (3) triagem para risco de broncoaspiração realizada pela equipe de Fonoaudiologia; (4) prescrição de suplemento nutricional oral em pacientes com via oral liberada.

O registro de complicações foi realizado por revisão de prontuário no momento da alta para determinar a ocorrência ou não de cada evento. O registro dos indicadores de processo foi realizado também no momento da alta para verificar o preenchimento ou não das metas de cuidado. Os desfechos de 30 dias (declínio funcional e reinternação precoce) foram determinados por meio de entrevistas telefônicas realizadas com o próprio paciente ou familiar responsável, em caso de déficit auditivo ou comprometimento cognitivo. Os profissionais que atuaram no registro de desfechos não estavam diretamente envolvidos nos processos de cuidado e foram os mesmos ao longo de todo o período do estudo. Os dados foram armazenados de forma segura na plataforma Research Eletronic Data Capture (REDCap, https://redcapbrasil.com.br/) e o estudo foi aprovado pelo Comitê de Ética em Pesquisa da Associação Beneficente Síria, Hospital do Coração (no 4.345.237).

\section{Análise de dados}

As variáveis categóricas foram descritas por meio de porcentagens e as variáveis intervalares usando-se a média e o desvio padrão. O teste exato de Fisher foi utilizado para comparar as variáveis categóricas entre os subgrupos. Os indicadores foram comparados entre os dois períodos (implantação versus consolidação) por meio de modelos logísticos multivariáveis com ajustes para idade, sexo, escolaridade, desempenho cognitivo (10-CS), grau de fragilidade (escala FRAIL) e motivo da internação. As análises foram ajustadas por estimativa robusta de variância, admitindo correlações intragrupos relacionadas a eventos que ferem o princípio de independência das observações, neste trabalho representadas por reinternações. Todas as análises foram realizadas com o software Stata 14.2 (https://www.stata.com) e um valor de p < 0,05 foi usado para definir diferenças estatisticamente significativas.

\section{Resultados}

No período entre janeiro de 2018 e dezembro de 2019, foram registradas no Programa Hospital Seguro para a Pessoa Idosa 1.738 internações de idosos frágeis. Foram excluídos da análise 320 indivíduos que tinham Índice de Barthel menor que 5 pontos, 148 que sofreram óbito durante a internação, 31 com tempo de permanência menor que 72 horas, 97 por dados faltantes no registro de desfechos intra-hospitalares, 33 que sofreram óbito nos 30 dias subsequentes à alta e 244 que não conseguimos contatar por telefone para a entrevista de 30 dias após a alta. Assim, foram incluídas na presente análise 865 internações de idosos frágeis, sendo 582 no período de implantação e 283 no de consolidação.

Os participantes apresentavam uma idade média de 81,9 $( \pm 8,6)$ anos e 53,9\% eram do sexo feminino. Quadros infecciosos (49,3\%) e doenças crônicas descompensadas (24\%) foram as principais causas de internação, e 92,8\% dos pacientes entraram no hospital pelo serviço de urgência e emergência. Embora nenhum critério de exclusão por causa de internação tenha sido utilizado, nota-se que a seleção de idosos frágeis determinou um amplo predomínio das causas clínicas. A principal causa cirúrgica foi representada pela fratura de quadril, presente em $16(1,8 \%)$ casos.

Os dados da avaliação multidimensional revelam que os critérios de elegibilidade adotados selecionaram uma população vulnerável e de alta complexidade, destacando-se que 49,7\% apresentavam comprometimento cognitivo, 75,6\% tinham alterações graves nas funções de membros inferiores e 97,5\% preenchiam critério para polifarmácia (uso de 5 ou mais medicamentos).

No período total analisado, 133 (15,4\%) participantes apresentaram declínio funcional persistente. Os fatores associados a um maior risco de declínio funcional foram a causa da internação (evento cardiovascular e queda com lesão determinando maior risco; $\mathrm{p}<0,001$ ), comprometimento nas funções 
de membros inferiores $(p=0,004)$, massa muscular reduzida $(p=0,017)$, sexo masculino $(p=0,019)$ e desnutrição $(\mathrm{p}=0,024)$. As características da amostra de acordo com o desfecho funcional estão descritas na Tabela 1.

\section{Tabela 1}

Características sociodemográficas e clínicas da amostra de acordo com o desfecho funcional da internação. São Paulo, Brasil, $2018 / 2019$ ( $n$ = 865).

\begin{tabular}{|c|c|c|c|c|}
\hline & $\begin{array}{l}\text { Amostra total } \\
\qquad \begin{array}{c}{[n=865]} \\
n(\%)\end{array}\end{array}$ & $\begin{array}{l}\text { Sem declínio funcional } \\
\qquad \begin{array}{c}\mathrm{n}=732] \\
\mathrm{n}(\%)\end{array}\end{array}$ & $\begin{array}{l}\text { Com declínio funcional } \\
\qquad \begin{array}{c}{[n=133]} \\
n(\%)\end{array}\end{array}$ & Valor de $p$ * \\
\hline \multicolumn{5}{|l|}{ Idade (anos) } \\
\hline $60-69$ & $84(9,7)$ & $75(10,3)$ & $9(6,8)$ & 0,067 \\
\hline $70-79$ & $222(25,7)$ & $192(26,2)$ & $30(22,6)$ & \\
\hline $80-89$ & $390(45,1)$ & $316(43,2)$ & $74(55,6)$ & \\
\hline$\geq 90$ & $169(19,5)$ & $149(20,4)$ & $20(15,0)$ & \\
\hline Sexo masculino & $398(46,1)$ & $324(44,4)$ & $74(55,6)$ & 0,018 \\
\hline Baixa escolaridade (< 8 anos) & $208(24,1)$ & $174(23,8)$ & $34(25,6)$ & 0,660 \\
\hline \multicolumn{5}{|l|}{ Arranjo de moradia antes da internação } \\
\hline Vivia sozinho(a) & $181(21,0)$ & $153(20,9)$ & $28(21,1)$ & 0,655 \\
\hline Com companheiro e/ou familiares & $654(75,7)$ & $555(75,9)$ & $99(74,4)$ & \\
\hline Institucionalizado(a) & $29(3,4)$ & $23(3,2)$ & $6(4,5)$ & \\
\hline Entrada pelo pronto-atendimento & $798(92,8)$ & $673(92,6)$ & $125(94,0)$ & 0,715 \\
\hline \multicolumn{5}{|l|}{ Motivo da internação } \\
\hline Quadro infeccioso & $426(49,3)$ & $366(50,1)$ & $60(45,1)$ & $<0,001$ \\
\hline Doença crônica descompensada & $207(24,0)$ & $185(25,3)$ & $22(16,5)$ & \\
\hline Evento cardiovascular & $45(5,2)$ & $32(4,4)$ & $13(9,8)$ & \\
\hline Queda com lesão & $39(4,5)$ & $25(3,4)$ & $14(10,5)$ & \\
\hline Outro & $147(17,0)$ & $123(16,8)$ & $24(18,1)$ & \\
\hline Comprometimento cognitivo $(10-\mathrm{CS} \leq 5)$ & $430(49,7)$ & $360(49,2)$ & $70(52,6)$ & 0,510 \\
\hline Delirium (definido pelo CAM) & $201(23,8)$ & $163(22,7)$ & $38(29,7)$ & 0,092 \\
\hline \multicolumn{5}{|l|}{ Força de preensão palmar ** } \\
\hline Preservada ( $\geq 30 \mathrm{~kg} / 20 \mathrm{~kg})$ & $120(15,3)$ & $105(15,7)$ & $15(12,9)$ & 0,572 \\
\hline Redução leve (26-9kg/16-9kg) & $104(13,3)$ & $91(13,6)$ & $13(11,2)$ & \\
\hline Redução grave ( $\leq 15 \mathrm{~kg} / 10 \mathrm{~kg})$ & $561(71,5)$ & $473(70,7)$ & $88(75,9)$ & \\
\hline \multicolumn{5}{|l|}{ Desempenho de membros inferiores (SPPB) } \\
\hline Normal (12-9 pontos) & $37(6,1)$ & $36(7,1)$ & $1(0,99)$ & 0,004 \\
\hline Alteração leve (8-5 pontos) & $111(18,3)$ & $99(19,6)$ & $12(11,9)$ & \\
\hline Alteração grave (4-0 pontos) & $458(75,6)$ & $370(73,3)$ & $88(87,1)$ & \\
\hline Perímetro da panturrilha reduzido $(<31 \mathrm{~cm})$ & $173(34,1)$ & $134(31,7)$ & $39(45,9)$ & 0,017 \\
\hline Desnutrição (MAN > 8) & $113(25,2)$ & $84(23,0)$ & $29(35,4)$ & 0,024 \\
\hline Recebendo nutrição enteral & $69(15,3)$ & $52(14,2)$ & $17(20,2)$ & 0,179 \\
\hline Lesão por pressão presente na admissão & $39(4,8)$ & $32(4,7)$ & $7(5,7)$ & 0,648 \\
\hline \multicolumn{5}{|l|}{ Número de medicações } \\
\hline $0-4$ & $15(2,5)$ & $13(2,5)$ & $2(2,1)$ & 0,599 \\
\hline $5-9$ & $172(28,2)$ & $149(29,0)$ & $23(24,2)$ & \\
\hline$\geq 10$ & $422(69,3)$ & $352(68,5)$ & $70(73,7)$ & \\
\hline
\end{tabular}

10-CS: 10-point Cognitive Screener; CAM: Confusion Assessment Method; MAN: Mini-Avaliação Nutricional (triagem); SPPB: Short Physical Performance Battery.

* Teste exato de Fisher;

** Valores de referência para homens e mulheres, respectivamente. 
A comparação dos indicadores de processo entre o período de implantação e o de consolidação revelou que o programa foi bem sucedido em melhorar os processos assistenciais fundamentais na qualidade do cuidado oferecido à pessoa idosa hospitalizada, incluindo reabilitação motora (de 74,1 para 84,3\%; p < 0,001), triagem de risco de broncoaspiração (de 38,5 para 82,8\%; p < 0,001) e suplementação nutricional oral (de 55,6 para 76,4\%; p < 0,001). A análise dos indicadores de processo está descrita na Tabela 2 .

A comparação dos indicadores de resultado entre o período de implantação e o de consolidação revelou que a taxa de declínio funcional persistente, que era 17,2\% no ano de implantação, caiu para $11,7 \%$ no ano de consolidação, com diferença significativa mesmo após o ajuste para potenciais vieses $(\mathrm{p}=0,009)$. A Figura 1 ilustra a evolução do indicador ao longo do período analisado em agrupamento bimestral.

Não encontramos diferenças significativas entre os dois períodos nos indicadores secundários, incluindo a incidência de quedas e lesão por pressão, como pode ser observado na Tabela 3.

\section{Discussão}

Neste estudo, avaliamos o impacto de um programa de melhoria dos processos assistenciais na atenção ao idoso hospitalizado, que foi planejado para a realidade dos hospitais brasileiros. Com uma estratégia estruturada em 10 passos, a implantação no prazo de um ano mostrou-se factível e o estudo revelou resultados promissores, com melhora significativa na maioria dos indicadores de processo e redução da taxa de declínio funcional persistente. Não encontramos na literatura outros programas amplos de melhoria da atenção ao idoso hospitalizado com efetividade demostrada nos países em desenvolvimento.

Não observamos padrão evolutivo de melhora em outros indicadores importantes, como taxa de quedas, lesão por pressão e permanência prolongada. Algumas hipóteses podem ser levantadas para explicar esse achado, como o fato de o hospital escolhido para o estudo já ter protocolos de segurança bem implementados, os indicadores dependerem de numerosos fatores que podem ter sido abordados de forma insuficiente e os eventos adversos monitorados terem apresentado frequência relativamente baixa.

Não observamos ainda redução nas taxas de reinternação precoce. Em uma metanálise que incluiu 30 ensaios clínicos, Facchinetti et al. 35 observaram que as intervenções específicas de continuidade do cuidado conseguiram reduzir reinternações precoces com risco relativo de 0,84 (IC95\%: 0,71-0,99).

Tabela 2

Indicadores de processo na comparação entre o período de implantação e o período de consolidação. São Paulo, Brasil, $2018 / 2019$ ( $n$ = 865).

\begin{tabular}{|c|c|c|c|}
\hline & $\begin{array}{c}\text { Período de } \\
\text { implantação } \\
\text { n (\%) }\end{array}$ & $\begin{array}{c}\text { Período de } \\
\text { consolidação } \\
\text { n (\%) }\end{array}$ & Valor de $p$ * \\
\hline $\begin{array}{l}\text { Rastreio de medicações inapropriadas pela equipe da } \\
\text { Farmácia dentro de } 72 \mathrm{~h}\end{array}$ & $482(82,8)$ & $234(82,7)$ & 0,651 \\
\hline $\begin{array}{l}\text { Reabilitação motora iniciada pela equipe de Fisioterapia } \\
\text { dentro de } 72 \mathrm{~h}\end{array}$ & $431(74,1)$ & $239(84,3)$ & $<0,001$ \\
\hline $\begin{array}{l}\text { Triagem para o risco de broncoaspiração realizada pela } \\
\text { equipe de Fonoaudiologia }\end{array}$ & $224(38,5)$ & $234(82,8)$ & $<0,001$ \\
\hline $\begin{array}{l}\text { Suplemento nutricional oral prescrito (em pacientes com } \\
\text { ingesta oral) }\end{array}$ & $323(55,5)$ & $216(76,4)$ & $<0,001$ \\
\hline
\end{tabular}

* Ajustado em modelo logístico multivariável para idade, sexo, escolaridade, desempenho cognitivo (rastreio cognitivo de 10 pontos), desempenho funcional prévio (Índice de Barthel), grau de fragilidade (escala FRAIL) e motivo da internação. 


\section{Figura 1}

Efeito do Programa Hospital Seguro para a Pessoa Idosa na taxa de declínio funcional associado à internação hospitalar. São Paulo, Brasil, 2018/2019 ( $\mathrm{n}=865)$.

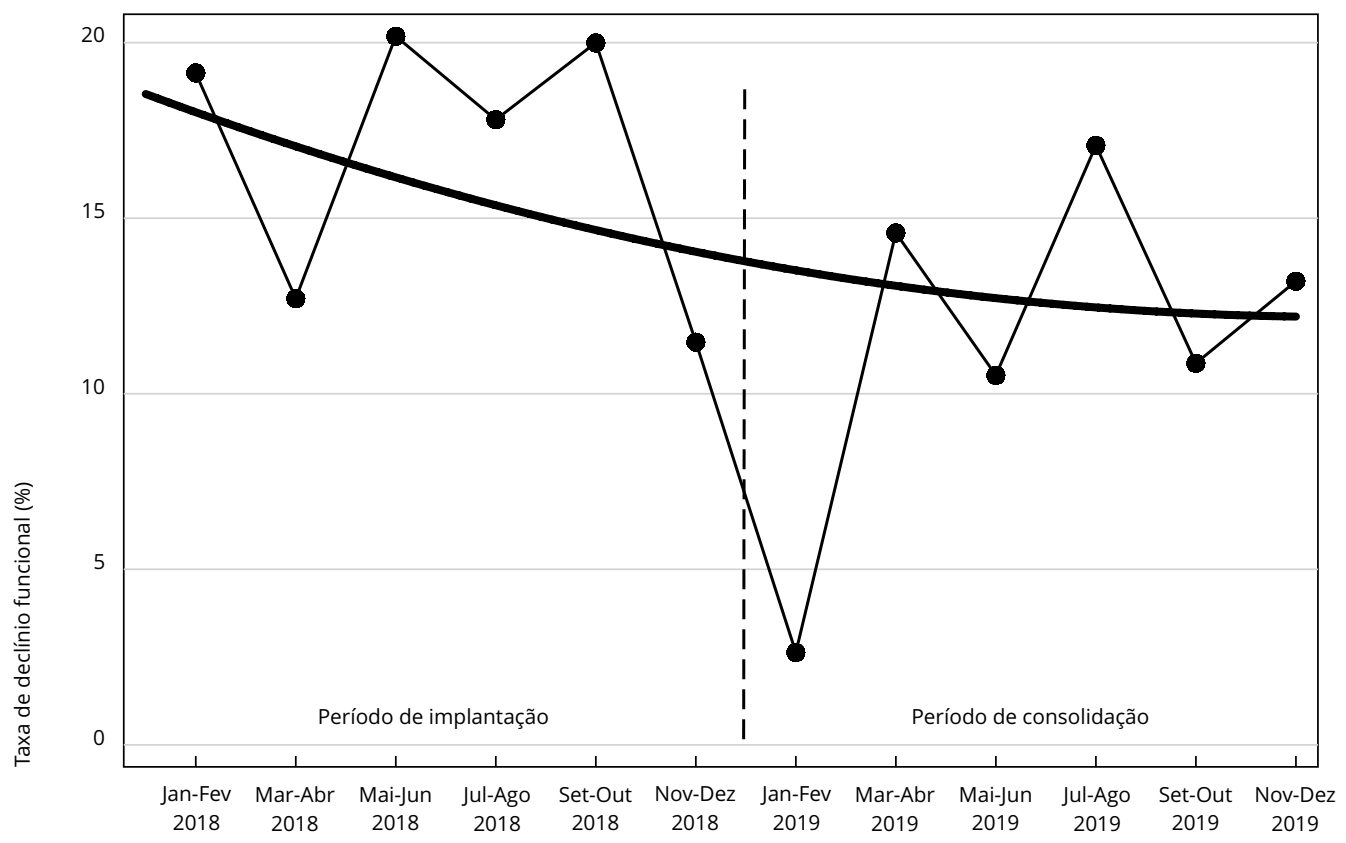

\section{Tabela 3}

Indicadores de resultado na comparação entre o período de implantação e o período de consolidação. São Paulo, Brasil, 2018/2019 (n = 865).

\begin{tabular}{|c|c|c|c|}
\hline & $\begin{array}{c}\text { Período de implantação } \\
\text { n (\%) }\end{array}$ & $\begin{array}{c}\text { Período de consolidação } \\
\text { n (\%) }\end{array}$ & Valor de $p$ * \\
\hline Declínio funcional ** & $100(17,2)$ & $33(11,7)$ & 0,009 \\
\hline Queda durante a internação & $12(2,1)$ & $8(2,9)$ & 0,269 \\
\hline Lesão por pressão incidente na internação *** & $13(2,2)$ & $3(1,1)$ & 0,286 \\
\hline Broncoaspiração durante a internação & $2(0,4)$ & $3(1,1)$ & 0,201 \\
\hline Transferência não planejada para a UTI & $56(9,7)$ & $21(7,5)$ & 0,285 \\
\hline Alta para instituição de longa permanência & $17(2,9)$ & $9(3,2)$ & 0,490 \\
\hline Internação prolongada (> 30 dias) & $51(8,8)$ & $35(12,4)$ & 0,333 \\
\hline Reinternação precoce ( $\leq 30$ dias) & $95(16,3)$ & $47(16,6)$ & 0,912 \\
\hline
\end{tabular}

UTI: unidade de terapia intensiva.

* Ajustado em modelo logístico multivariável para idade, sexo, escolaridade, desempenho cognitivo (rastreio cognitivo de 10 pontos), desempenho funcional prévio (Índice de Barthel), grau de fragilidade (escala FRAIL) e motivo da internação;

** Declínio funcional definido como redução de 5 ou mais pontos no Índice de Barthel na comparação entre o funcionamento prévio à internação e 30 dias após a alta;

*** Consideradas as lesões com estágio 2 ou maiores. 
Embora o aspecto da transição do cuidado tenha sido contemplado no passo 9 do Programa, é provável que as ações não tenham apresentado intensidade suficiente para modificar o desfecho. Assim, em futuras implantações deve-se considerar o fortalecimento desse componente com ações específicas e bem planejadas.

Em um hospital universitário brasileiro, Carvalho et al. 36 observaram que 28,1\% dos idosos hospitalizados não haviam recuperado o desempenho em atividades básicas da vida diária 30 dias após a alta. Uma metanálise encontrou valores semelhantes de declínio funcional em idosos frágeis hospitalizados, com taxas variando entre 20 e $40 \%$ na maioria dos estudos 13 . Em nosso trabalho, a taxa de declínio funcional caiu de 17,1\% para 11,7\%, revelando que o programa tem potencial de benefício mesmo em instituições que já registram taxas baixas do desfecho. É possível que o benefício seja maior nas instituições que apresentam taxas mais elevadas, hipótese a ser testada em estudos futuros.

O declínio funcional associado à hospitalização entre os idosos é um fenômeno amplamente negligenciado. Entre os fatores que determinam a subvalorização desse problema podemos citar o fato de que o ônus da dependência funcional não é absorvido pelo hospital, mas transferido ao idoso e seus familiares no momento da alta, fazendo com que haja pouco ou nenhum esforço das instituições para evitar essa complicação. O problema é potencializado pela cultura fatalista segundo a qual o declínio funcional é inerente ao processo de adoecimento do idoso. Ocorre ainda que muitos hospitais em processo de acreditação passaram a implementar protocolos de segurança equivocados, nos quais a principal estratégia para prevenir as quedas é a restrição coercitiva da mobilidade dos pacientes idosos 37.

Um estudo realizado com acelerômetros revelou que idosos hospitalizados capazes de deambular passam $83 \%$ do tempo deitados na cama durante uma internação 38. Após 5 dias de mobilidade reduzida, o idoso perde em média $4 \%$ da sua massa magra e já se observa redução nas medidas de força 39 . Os recursos de fisioterapia no ambiente hospitalar geralmente são escassos e muitas vezes voltados apenas para o atendimento de condições respiratórias. São poucos os hospitais que contam com o apoio de educadores físicos ou terapeutas ocupacionais em suas equipes. Os protocolos multidisciplinares de promoção da mobilidade e os programas de mobilização baseados em voluntários da comunidade ainda são raros. Assim, são necessários novos estudos para trazer o problema à tona de forma mais clara e propor soluções com boa relação de custo-efetividade.

É provável que a redução da taxa de declínio funcional observada neste trabalho tenha resultado de uma maior aplicação de recursos de reabilitação precoce e suplementação alimentar. Há boas evidências de que a estratégia de identificar pacientes frágeis e oferecer programas de exercício físico associados a um aporte calórico-proteico adequado possa reduzir o impacto funcional da internação 14 . Com base em uma revisão sistemática, Ley et al. 40 estimaram que idosos hospitalizados devem exercitar-se por 20 minutos pelo menos duas vezes ao dia para evitar o declínio funcional. No nosso estudo os idosos frágeis receberam, por protocolo, fisioterapia motora diária com frequência de uma a duas vezes ao dia, mas não foram obtidos registros exatos quanto à frequência e a duração dos exercícios.

Este trabalho reúne algumas qualidades que merecem ser ressaltadas. Em primeiro lugar, conseguiu um tamanho amostral suficiente para detectar diferenças estatisticamente significativas em indicadores importantes. Em segundo, foi realizado em um cenário naturalístico, sem financiamento externo, com recursos do próprio hospital, reforçando a factibilidade da proposta. Em terceiro lugar, embora o planejamento e a consultoria tenham sido realizados por especialistas na área de envelhecimento, a implantação foi feita por uma equipe não especializada em um hospital que não conta com equipe de Geriatria, em um cenário compatível com o da maioria dos hospitais do SUS. Em quarto, os desfechos foram registrados de forma sistematizada com uma metodologia que foi mantida ao longo de todo o estudo, atenuando assim a possibilidade de potenciais vieses.

A principal limitação do trabalho deve-se ao próprio delineamento observacional e à ausência de um grupo controle. Consideramos que um estudo controlado seria inviável dentro de um único hospital, já que a proposta do programa é justamente proporcionar modificações disseminadas que permeiam todo o percurso do paciente idoso no hospital. Em oportunidades futuras, a possibilidade de um ensaio clínico multicêntrico com metodologia de cluster deve ser considerada.

Outra limitação a ser mencionada é o fato de que os indicadores de interesse começaram a ser monitorados de forma concomitante à implantação do programa. É possível que algumas melhorias conseguidas no primeiro ano não tenham sido detectadas como mudança, reduzindo assim a dimensão do impacto encontrado. Reconhecemos ainda como limitação o fato de apenas 49,7\% do total 
de internações no período terem sido incluídas na análise final. Embora tenham sido necessários para garantir a qualidade do estudo, os critérios de exclusão podem ter limitado a generalização dos achados. Ressaltamos ainda a perda de $5,6 \%$ do total de internações por dados faltantes e de $14 \%$ por impossibilidade de contato telefônico, perdas que podem ter introduzido alguns vieses.

Um dos propósitos fundamentais deste projeto foi o desenvolvimento de um modelo que pudesse ser compatível com os recursos estruturais e humanos presentes no maior número possível de hospitais brasileiros. Entre os elementos planejados com essa intenção podemos citar a estratégia de implantação disseminada (em vez da construção de uma unidade específica), o aproveitamento dos profissionais disponíveis (em vez da contratação de profissionais especializados em Geriatria e Gerontologia) e a possibilidade de adaptações para a realidade local. No entanto, é preciso ressaltar que a implantação depende de pré-requisitos básicos, como o apoio estratégico da direção do hospital, a adesão das lideranças assistenciais, a disponibilidade de profissionais assistenciais em número adequado, a cultura de protocolos gerenciados e a estrutura básica para o monitoramento de indicadores. Nos hospitais com carência de recursos básicos, naqueles com cultura de segurança do paciente fraca ou nos quais a atenção ao idoso não é uma prioridade estratégica, a tentativa de implantar esse programa pode ser infrutífera.

Neste momento, o Programa Hospital Seguro para a Pessoa Idosa está sendo implantado em quatro hospitais de diferentes estados brasileiros em um projeto-piloto financiado pelo PROADI-SUS. Esperamos que experiência obtida nesses centros sirva para aprimorar o programa e forneça novos elementos para planejar a disseminação da iniciativa.

Em conclusão, desenvolvemos um programa de melhoria da atenção ao idoso hospitalizado com metodologia de implantação sistematizada e que parece ser factível para a realidade de muitos hospitais brasileiros. Os resultados preliminares obtidos neste estudo parecem promissores e a estratégia de disseminação dessa iniciativa na rede hospitalar deve ser considerada no planejamento das políticas de saúde pública dessa área. 


\section{Colaboradores}

D. Apolinario contribuiu no delineamento do estudo, análise de dados e composição do manuscrito. S. T. F. Yamaguti e E. M. S. Lara contribuíram no delineamento do estudo e composição do manuscrito. A. F. Dutra contribuiu no delineamento do estudo, coleta de dados e composição do manuscrito. R. C. P. Coli e B. Weber contribuíram no delineamento do estudo e revisão do manuscrito. Todos os autores aprovaram a versão final do artigo para publicação.

\section{Informações adicionais}

ORCID: Daniel Apolinario (0000-0002-0261129X); Siomara Tavares Fernandes Yamaguti (00000002-0617-0372); Adriana Fatina Dutra (00000002-0403-1993); Enilda Maria de Sousa Lara (0000-0001-7249-620X); Rita de Cassia Pires Coli (0000-0001-6416-4029); Bernardete Weber (00000003-1912-652X).

\section{Referências}

1. Instituto Brasileiro de Geografia e Estatística. Pesquisa Nacional por Amostra de Domicílios Contínua. https://sidra.ibge.gov.br/tabe la/6407 (acessado em 25/Ago/2020).

2. Departamento de Informática do SUS. Sistema de Informações Hospitalares do SUS. http:// tabnet.datasus.gov.br/cgi/tabcgi.exe?sih/cnv/ niuf.def (acessado em 25/Ago/2020).

3. Garcez-Leme LE, Leme MD. Costs of elderly health care in Brazil: challenges and strategies. MedicalExpress (São Paulo) 2014; 1:3-8.

4. Mitnitski AB, Mogilner AJ, Rockwood K. Accumulation of deficits as a proxy measure of aging. ScientificWorldJournal 2001; 1:323-36.

5. Costa CS, Flores TR, Wendt A, Neves RG, Tomasi E, Cesar JA, et al. Inequalities in multimorbidity among elderly: a population-based study in a city in Southern Brazil. Cad Saúde Pública 2018; 34:e00040718.

6. Fried LP, Tangen CM, Walston J, Newman AB, Hirsch C, Gottdiener J, et al. Frailty in older adults: evidence for a phenotype. J Gerontol A Biol Sci Med Sci 2001; 56:M146-56.

7. Hartley P, Adamson J, Cunningham C, Embleton G, Romero-Ortuno R. Clinical frailty and functional trajectories in hospitalized older adults: a retrospective observational study. Geriatr Gerontol Int 2017; 17:1063-8.

8. Santos GAS, Boing AC. Mortalidade e internações hospitalares por intoxicações e reações adversas a medicamentos no Brasil: análise de 2000 a 2014. Cad Saúde Pública 2018; 34:e00100917.

9. Clark S, Shaw C, Padayachee A, Howard S, Hay K, Frakking TT. Frailty and hospital outcomes within a low socioeconomic population. QJM 2019; 112:907-13.

10. Bredthauer D, Becker C, Eichner B, Koczy P, Nikolaus T. Factors relating to the use of physical restraints in psychogeriatric care: a paradigm for elder abuse. Z Gerontol Geriatr 2005; 38:10-8

11. Brown CJ, Friedkin RJ, Inouye SK. Prevalence and outcomes of low mobility in hospitalized older patients. J Am Geriatr Soc 2004; 52:1263-70.

12. Zisberg A, Shadmi E, Gur-Yaish N, Tonkikh O, Sinoff G. Hospital-associated functional decline: the role of hospitalization processes beyond individual risk factors. J Am Geriatr Soc 2015; 63:55-62.

13. Cunha AIL, Veronese N, Melo Borges S, Ricci NA. Frailty as a predictor of adverse outcomes in hospitalized older adults: a systematic review and meta-analysis. Ageing Res Rev 2019; 56:100960.

14. Martínez-Velilla N, Casas-Herrero A, Zambom-Ferraresi F, Asteasu MLS, Lucia A, Galbete $\mathrm{A}$, et al. Effect of exercise intervention on functional decline in very elderly patients during acute hospitalization: a randomized clinical trial. JAMA Intern Med 2019; 179:28-36. 
15. Resnick B, Boltz M. Optimizing function and physical activity in hospitalized older adults to prevent functional decline and falls. Clin Geriatr Med 2019; 35:237-51.

16. Inouye SK, Bogardus ST Jr, Baker DI, LeoSummers L, Cooney LM Jr. The Hospital Elder Life Program: a model of care to prevent cognitive and functional decline in older hospitalized patients. Hospital Elder Life Program. J Am Geriatr Soc 2000; 48:1697-706.

17. Hshieh TT, Yang T, Gartaganis SL, Yue J, Inouye SK. Hospital Elder Life Program: systematic review and meta-analysis of effectiveness. Am J Geriatr Psychiatry 2018; 26:1015-33.

18. Capezuti EA, Bricoli B, Boltz MP. Nurses improving the care of healthsystem elders: creating a sustainable business model to improve care of hospitalized older adults. J Am Geriatr Soc 2013; 61:1387-93.

19. Hansen LO, Greenwald JL, Budnitz T, Howell E, Halasyamani L, Maynard G, et al. Project BOOST: effectiveness of a multihospital effort to reduce rehospitalization. J Hosp Med 2013; 8:421-7.

20. Fulmer T, Mate KS, Berman A. The age-friendly health system imperative. J Am Geriatr Soc 2018; 66:22-4.

21. World Health Organization. The conceptual framework for the International Classification for Patient Safety. (Final Technical Report, 2009). https://www.who.int/patientsafety/ taxonomy/icps_full_report.pdf (acessado em 25/Ago/2021).

22. Veras R. Linha de cuidado para o idoso: detalhando o modelo. Rev Bras Geriatr Gerontol 2016; 19:887-905.

23. Oliveira M, Silveira DP, Neves R, Veras, R, Estrella K, Assalim VM, et al. Idoso na saúde suplementar: uma urgência para a saúde da sociedade e para a sustentabilidade do setor. Rio de Janeiro: Agência Nacional de Saúde Suplementar; 2016.

24. Leis JA, Shojania KG. A primer on PDSA: executing plan-do-study-act cycles in practice, not just in name. BMJ Qual Saf 2017; 26:572-7.

25. Morley JE, Malmstrom TK, Miller DK. A simple frailty questionnaire (FRAIL) predicts outcomes in middle aged African Americans. J Nutr Health Aging 2012; 16:601-8.

26. Aprahamian I, Lin SM, Suemoto CK, Apolinario D, Cezar NOC, Elmadjian SM, et al. Feasibility and factor structure of the FRAIL scale in older adults. J Am Med Dir Assoc 2017; 18:367.e11-367.e18.

27. Mahoey FI, Barthel DW. Functional evaluation: the Barthel Index. Md State Med J 1965; 14:61-5.

28. Apolinario D, Lichtenthaler DG, Magaldi RM, Soares AT, Busse AL, Amaral JR, et al. Using temporal orientation, category fluency, and word recall for detecting cognitive impairment: the 10-point cognitive screener (10-CS). Int J Geriatr Psychiatry 2016; 31:4-12.
29. Inouye SK, van Dyck CH, Alessi CA, Balkin S, Siegal AP, Horwitz RI. Clarifying confusion: the confusion assessment method. A new method for detection of delirium. Ann Intern Med 1990; 113:941-8.

30. Guralnik JM, Simonsick EM, Ferrucci L, Glynn RJ, Berkman LF, Blazer DG, et al. A short physical performance battery assessing lower extremity function: association with self-reported disability and prediction of mortality and nursing home admission. J Gerontol 1994; 49:M85-94.

31. Rubenstein LZ, Harker JO, Salvà A, Guigoz Y, Vellas B. Screening for undernutrition in geriatric practice: developing the Short-Form Mini-Nutritional Assessment (MNA-SF). J Gerontol A Biol Sci Med Sci 2001; 56:M366-72.

32. Collin C, Wade DT, Davies S, Horne V. The Barthel ADL Index: a reliability study. Int Disabil Stud 1988; 10:61-3.

33. Hsieh YW, Wang CH, Wu SC, Chen PC, Sheu CF, Hsieh CL. Establishing the minimal clinically important difference of the Barthel Index in stroke patients. Neurorehabil Neural Repair 2007; 21:233-8.

34. By the 2019 American Geriatrics Society Beers Criteria Update Expert Panel. American Geriatrics Society 2019 Updated AGS beers criteria for potentially inappropriate medication use in older adults. J Am Geriatr Soc 2019; 67:674-94.

35. Facchinetti G, D’Angelo D, Piredda M, Petitti T, Matarese M, Oliveti A, De Marinis MG. Continuity of care interventions for preventing hospital readmission of older people with chronic diseases: a meta-analysis. Int J Nurs Stud 2020; 101:103396.

36. Carvalho TC, Valle AP, Jacinto AF, Mayoral VFS, Villas-Boas PJF. Impacto da hospitalização na funcionalidade de idosos: estudo de coorte. Rev Bras Geriatr Gerontol 2018; 21:134-42.

37. Growdon ME, Shorr RI, Inouye SK. The tension between promoting mobility and preventing falls in the hospital. JAMA Intern Med 2017; 177:759-60.

38. Brown CJ, Redden DT, Flood KL, Allman RM. The underrecognized epidemic of low mobility during hospitalization of older adults. J Am Geriatr Soc 2009; 57:1660-5.

39. Tanner RE, Brunker LB, Agergaard J, Barrows KM, Briggs RA, Kwon OS, et al. Age-related differences in lean mass, protein synthesis and skeletal muscle markers of proteolysis after bed rest and exercise rehabilitation. J Physiol 2015; 593:4259-73.

40. Ley L, Khaw D, Duke M, Botti M. The dose of physical activity to minimise functional decline in older general medical patients receiving 24-hr acute care: a systematic scoping review. J Clin Nurs 2019; 28:3049-64. 


\section{Abstract}

Hospitalized elderly persons experience increased risk of complications and functional decline. The current study assessed the results of the Safe Hospital Program for the Elderly Person. The intervention was conducted in a charitable hospital in the city of São Paulo, Brazil, using a 10-step structured implementation strategy, including screening for frailty, multidimensional evaluation, unique treatment plan, implementation of protocols, and discharge management strategies. The study analyzed 865 hospitalizations of frail elders over the course of two years. Process and results indicators were compared between the first year (implementation) and second year (consolidation). Comparison of the indicators between the two periods showed that the program improved fundamental processes in care for this population, including motor rehabilitation initiated in the first 72 hours (74.1 vs. 84.3\%; $p<0.001$ ), screening for risk of bronchial aspiration (38.5 vs. 82.8\%; $p<0.001$ ), and oral nutritional supplementation (55.6 vs. 76.4\%; $p<0.001$ ). The functional decline rate, which was $17.2 \%$ in the implementation year, decreased to $11.7 \%$ in the consolidation year, with a significant difference after adjusting in the multivariate model $(p=0.009)$. In conclusion, we developed a feasible program for the reality of Brazilian hospitals and that can be reproduced in other centers through a systematized implementation methodology. The results pertaining to patient care indicators and clinical outcomes appear promising. This initiative's dissemination should be considered in public health policy planning for the hospital network.

Hospitalization; Frailty; Activities of Daily Living; Quality Improvement; Aged

\section{Resumen}

Al ser hospitalizado, un anciano presenta mayor riesgo de complicaciones y deterioro funcional. El presente estudio evaluó los resultados del Programa Hospital Seguro para Personas Ancianas. La intervención se realizó en un hospital filantrópico de la ciudad de São Paulo, Brasil, a través de una estrategia de implementación estructurada en 10 pasos, incluyendo rastreo de debilidad, evaluación multidimensional, plan terapéutico singular, implementación de protocolos y estrategias de gestión de alta. Se analizaron a lo largo de dos años 865 internamientos de ancianos débiles. Se compararon indicadores de proceso y resultado entre el primer año (período de implementación) y el segundo año (período de consolidación). La comparación de los indicadores entre los dos periodos reveló que el programa mejoró procesos asistenciales fundamentales en la calidad del cuidado para esa población, incluyendo rehabilitación motora iniciada en las primeras 72 horas $(74,1$ vs. 84,3\%; $p<0,001)$, evaluación del riesgo de broncoaspiración (38,5 vs. 82, 8\%; $p<0,001)$ y suplementación nutricional oral $(55,6$ vs. 76,4\%; $p<0,001)$. La tasa de deterioro funcional, que era $17,2 \%$ en el año de implementación, cayó a 11,7\% en el año de consolidación, con una diferencia significativa tras el ajuste en el modelo multivariable $(p=0,009)$. En conclusión, desarrollamos un programa factible para la realidad de los hospitales brasileños, que puede ser reproducido en otros centros a través de una metodología sistematizada de implementación. Los resultados relacionados con los indicadores de procesos asistenciales y resultados clínicos parecen prometedores. La difusión de esta iniciativa debe ser considerada en la planificación de las politicas de salud pública para la red hospitalaria.

Hospitalización; Fragilidad; Actividades

Cotidianas; Mejoramiento de la Calidad; Anciano
Recebido em 22/Out/2020

Versão final reapresentada em 08/Mai/2021

Aprovado em 09/Ago/2021 